\title{
RESONANT ABSORPTION IN A PLASMA STEP PROFILE
}

\author{
E. Ahedo and J. R. Sanmartín \\ Escuela Técnica Superior de Ingenieros Aeronáuticos, Universidad Politécnica de Madrid, 28040-Madrid, \\ Spain \\ (Received 19 June 1986; and in revised form 28 October 1986)
}

\begin{abstract}
Resonant absorption of $p$-polarized light shined on a plane-layered plasma with a step profile, is discussed as a function of wavelength (or critical density $n_{c}$ ) of the light; for simplicity the incidence angle is assumed small. If $n_{c}$ lies within or above the step, the absorption $A$ is given by Ginzburg's result modified by strong reflections at the foot and top of the step. The absorption above is total for particular values of $n_{c}$ and 0 . For $n_{c}$ crossing the top of the density step the absorption is not monotonical: it exhibits a minimum that vanishes for zero radius of curvature $\sigma$ there $\left(A \sim|\ln \sigma|^{-2}\right)$, and zero collision frequency $v\left(A \sim|\ln y|^{-2}\right)$. The results are applied to the profile produced by irradiating a solid target with a high-intensity pulse that steepens the plasma by radiation pressure.
\end{abstract}

\section{INTRODUCTION}

$P$-POLARIZED light incident at angle $\theta$ on a plane layered plasma is known to experience resonant absorption at the critical density $n_{c}(\omega)$, where the light frequency $\omega$ and the local plasma frequency are equal (GINZBURG, 1970). For a cold, collisionless plasma with a gentle, linear density profile the absorption $A$ of low intensity light is a function of just one parameter: $A=A_{G}(q)$ with $q \equiv(L / \hat{\tau})^{2 / 3} \sin ^{2} \theta$, where $\hat{z}=c / \omega$ and $L \equiv n_{c} /$ (density gradient). Absorption is at most $50 \%$, the maximum occurring at $q \simeq 0.5$ (FoRsLUND et al., 1975).

If the profile is only piece-wise linear, total or near total absorption may take place under some stringent conditions. Aliev et al. (1977) considered a density step: a thin layer of sharp density gradient with $L$ comparable to $\hat{k}$, the plasma being gently inhomogeneous at higher and lower densities; he assumed $n_{c}$ to lie within the step, and $n_{c} \cos ^{2} \theta$, where reflection occurs, below it. KINDEL et al. (1975) analysed a double step, and KULL (1983) a high-density background coated with a thin layer.

Here we consider how absorption in a single step depends on the light frequency; for simplicity $\theta$ is assumed to be small. We let the critical density for the light range from within to above the step. We find that if the reflections occurring there, and at the region around critical, are matched, there is total absorption above; specific values of both $n_{c}$ and $\theta$ are required, however, for this result. On the other hand, the absorption is found to exhibit a sharp minimum just at the top of the step independently of $\theta$. That simple feature, apparently paradoxical because the density gradient is monotonical and $A_{G}(q)$ has no minimum, could be used, we suggest, as a diagnostics tool; probing $p$-polarized light, at a few frequencies and a weak enough intensity (so as not to affect the profile) should be shined on the plasma and $A$ measured.

In Section 2 we calculate absorption within and above the step; in Section 3 we study in detail absorption around the top. In Section 4 we apply this analysis to a particular plasma profile. Results are discussed in Section 5. 


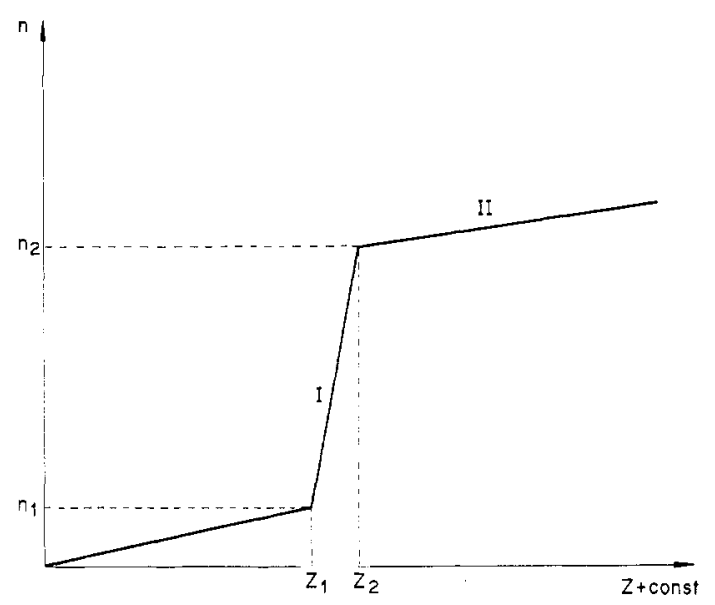

FIG. 1.- Model of plasma step profile. $P$-polarized light is incident from the left at small incidence angle. The critical surface is considered to lie in either region I or II, depending on wave frequency $\omega \equiv c / \hbar$.

\section{ABSORPTION IN A PROFILE STEP}

Consider the plasma profile of Fig. 1, stratified along the $z$-axis, and let $p$-polarized light of frequency $\omega=c / t$ be incident on the plasma at a small angle $\theta$. Reflection occurs at $n_{c} \cos ^{2} \theta$, very close to the critical density $n_{c}$ where absorption takes place. For each straight piece of the profile and for each wavelength $\hat{t}$ we can define a length $L \equiv n_{\mathrm{c}}(\hat{\pi}) /(\mathrm{d} n / \mathrm{d} z)$ so that the dielectric function takes the form

$$
\varepsilon(z, \hat{\pi}) \simeq 1-\frac{n}{n_{c}}+\mathrm{i} \frac{v}{\omega} \equiv \frac{-z+\text { const }}{L}+\mathrm{i} \frac{\nu}{\omega} .
$$

Thus $L^{-1}=|\mathrm{d} \varepsilon / \mathrm{d} z|$. The imaginary part of $\varepsilon$, due to a small collision frequency $v$, is neglected except where needed to take care of a singularity at $n=n_{c}$.

We consider here absorption in regions I and II, with $n_{c} / n_{2}$ of order unity. We assume $L / \hat{\pi}$ of order unity for region I and large for region II. Since $L_{\mathrm{I}}$ is comparable to $\lambda$ and since the slope changes suddenly at points 1 and 2, usual results (GinzBuRG, 1970; ForsLund et al., 1975) are invalid. Thus absorption is not just $A_{G}$ and a self-consistent calculation is needed (for $n_{c}<n_{1}$, i.e. $\varepsilon_{1}<0$, we trivially have $A=A_{G}$ ). It suffices to study the magnetic field, which is perpendicular to the plane of incidence and may be written as $\operatorname{Re}\left[B_{x}(z) \times \exp \left(\mathrm{i} \hat{t}^{-1} y \sin \theta-\mathrm{i} \omega t\right)\right] ; B_{x}$ is a complex quantity determined by the equation

$$
\frac{\mathrm{d}^{2} B_{x}}{\mathrm{~d} z^{2}}-\frac{1}{\varepsilon} \frac{\mathrm{d} \varepsilon}{\mathrm{d} z} \frac{\mathrm{d} B_{x}}{\mathrm{~d} z}+\frac{\varepsilon-\sin ^{2} \theta}{\hbar^{2}} B_{x}=0 .
$$

Absorption in region $\mathrm{I}\left(\mathrm{n}_{1}<\mathrm{n}_{c}<\mathrm{n}_{2}\right)$.

Since $L_{1} \sim \lambda$ and $\theta \ll 1$ we have $q_{\mathrm{I}} \equiv\left(L_{\mathrm{l}} / \tilde{z}\right)^{2 / 3} \sin ^{2} \theta$ small. This suggests that absorption in region I should be low; the simplest way to determine it is then to use 
a standard formula for absorbed energy per unit time and area (LANDAU and LIFSHITZ, 1960), which here reads

$$
\dot{E}_{a}(\text { absorbed })=\int \frac{v \mathrm{~d} z}{8 \pi|\varepsilon|^{2}}\left|B_{x}\right|^{2} \sin ^{2} \theta\left[1+\frac{\hbar^{2}}{\sin ^{2} \theta}\left|\frac{\mathrm{d} \ln B_{x}}{\mathrm{~d} z}\right|^{2}\right] .
$$

In the limit $v / \omega \rightarrow 0$, and setting $1-n / n_{c}=-z / L_{1}$, we get $v /|\varepsilon|^{2} \rightarrow \pi \omega \delta\left(z / L_{1}\right)$. In addition, the profile being linear around $n_{c}$, one may show that the bracket in (3) behaves as $1+\left[z \hat{t}^{-1} \sin \theta \ln \left(z \hat{t}^{-1} \sin \theta\right)\right]^{2}$ for $z / t \rightarrow 0$ (LANDAU and LifshiTz, 1960). Equation (3) then becomes $\dot{E}_{a}=\left.\left(L_{\mathrm{l}} / 8 \dot{\pi}_{\mathrm{r}}\right) B_{c}\right|^{2} \sin ^{2} \theta$, where $B_{c} \equiv B_{x}(0)$. If $\left|B_{\infty}\right|$ is the amplitude of the incident magnetic field in vacuum, we have

$$
A=\pi q_{1}\left(L_{\mathrm{l}} / \hat{\kappa}\right)^{1 / 3}\left|B_{c} / B_{x}\right|^{2}
$$

We may now determine $B_{c} / B_{x}$ as if there was no absorption, that is, we may neglect $q_{1}$ and $v / \omega$.

Within the step, equation (2) becomes

$$
\frac{\mathrm{d}^{2} B_{x}}{\mathrm{~d} \xi^{2}}-\frac{1}{\xi} \frac{\mathrm{d} B_{x}}{\mathrm{~d} \xi}+\xi B_{x}=q_{1} B_{x} \simeq 0
$$

whose solution is

$$
B_{x}=B_{c} \frac{A \mathrm{i}^{\prime}(-\xi)+h B \mathrm{i}^{\prime}(-\xi)}{A \mathrm{i}_{0}^{\prime}+h B \mathrm{i}_{0}^{\prime}}
$$

Here $h$ is a constant, $\xi \equiv\left(L_{1} / \not\right)^{2 / 3} \varepsilon, A \mathrm{i}_{0}^{\prime} \equiv A \mathrm{i}^{\prime}(0), B \mathrm{i}_{0}^{\prime} \equiv B \mathrm{i}^{\prime}(0)$ and $A \mathrm{i}^{\prime}, B \mathrm{i}^{\prime}$ are the derivatives of Airy functions (AnTOSIEwICZ, 1964). Below point 1 the field consists of incoming and outgoing WKB waves:

$$
B_{x}=B_{\infty} \varepsilon^{1 / 4}\left[\exp \left(-\mathrm{i} \int_{z}^{z_{1}} \varepsilon^{1 / 2} \mathrm{~d} z / \lambda\right)+R \exp \left(\mathrm{i} \int_{z}^{z_{1}} \varepsilon^{1 / 2} \mathrm{~d} z / \lambda\right)\right] .
$$

Continuity of dln $B_{x} / \mathrm{d} z$ at point 1 gives

$$
R=\left(\alpha_{1}^{\dagger}+h \beta_{1}^{\dagger}\right) /\left(\alpha_{1}+h \beta_{1}\right)
$$

where

$$
\alpha(\xi) \equiv A \mathrm{i}^{\prime}(-\xi)+\mathrm{i} \xi^{1 / 2} A \mathrm{i}(-\xi), \quad \beta(\xi) \equiv B \mathrm{i}^{\prime}(-\xi)+\mathrm{i} \xi^{1 / 2} B \mathrm{i}(-\xi)
$$

and $\alpha_{1} \equiv \alpha\left(\xi_{1}\right), \alpha_{1}^{\dagger}$ is its complex conjugate, and so on. If $h$ is real (as found below), one gets $|R|=1$, in agreement with our computing $B_{c} / B_{\infty}$ as if there was no absorption. Next, continuity of $B_{x}$ at 1 , yields $B_{c} / B_{\infty}$ and thus $A$ in (4):

$$
A=A_{G}\left(q_{\mathrm{I}}\right) \frac{\left(1-3^{1 / 2} h\right)^{2} \xi_{1}^{1 / 2} / \pi}{\left(A \mathrm{i}_{1}^{\prime}+h B \mathrm{i}_{1}^{\prime}\right)^{2}+\xi_{1}\left(A \mathrm{i}_{1}+h B \mathrm{i}_{1}\right)^{2}} \ll 1 .
$$


We used $B \mathrm{i}_{0}^{\prime} / A \mathrm{i}_{0}^{\prime}=-3^{1 / 2}$, and $4 \pi^{2}\left|A \mathrm{i}_{0}^{\prime}\right|^{2} q \simeq A_{G}(q)$ for $q$ small; note also that $\left|\mathrm{Ai}_{0}^{\prime}\right|^{2} \simeq 0.067$.

To find $h$ we require continuity of $\mathrm{d} \ln B_{x} / \mathrm{d} z$ at point 2 ; using (6) we get

$$
\begin{aligned}
& h=-\frac{A i_{2}^{\prime}+\left(\tilde{\hbar} / L_{1}\right)^{1 / 3} \xi_{2} A i_{2} / D}{B i_{2}^{\prime}+\left(\hat{z} / L_{1}\right)^{1 / 3} \xi_{2} B i_{2} / D}, \\
& D \equiv \hat{\hbar} \operatorname{dln} B_{x} /\left.\mathrm{d} z\right|_{z_{2}} \cdot
\end{aligned}
$$

Beyond $z_{2}$ the wave is evanescent; since $L_{\mathrm{ll}} / \hat{\pi}$ is large, the wave may be written in WKB form (as long as $\varepsilon_{2}$ is not close to zero): $B_{x} \propto|\varepsilon|^{1 / 4} \times \exp$ $\left(-\int_{z_{2}}^{z}|\varepsilon|^{1 / 2} \mathrm{~d} z / \hat{\pi}\right)$. Thus

$$
D \simeq-\left|\varepsilon_{2}\right|^{1 / 2}, \quad h \simeq-\frac{A \mathrm{i}_{2}^{\prime}+\left|\xi_{2}\right|^{1 / 2} A \mathrm{i}_{2}}{B \mathrm{i}_{2}^{\prime}+\left|\xi_{2}\right|^{1 / 2} B \mathrm{i}_{2}}
$$

Equations (10) and (13) yield $A\left(q_{1}, \xi_{1}, \xi_{2}\right)$. Note that smoothing the step (setting $\mathrm{d} n / \mathrm{d} z \rightarrow 0$ in I) and letting $\theta \rightarrow 0$ appropriately, we have $\xi_{1},\left|\xi_{2}\right| \rightarrow \infty$ and $q_{1}$ fixed; using next the asymptotic behaviour of the Airy functions, we first get $h \rightarrow 0$ and then $A \rightarrow A_{G}\left(q_{1}\right)$ in (10). The limit of this same equation for the opposite case, $\mathrm{d} n / \mathrm{d} z \rightarrow \infty$ in $I$ or $L_{\mathrm{l}} / \hat{\pi} \rightarrow 0$ (a density jump), may be easily obtained too; this case was studied by KLLL (1983) for arbitrary $\theta$.

Absorption in region II $\left(\mathrm{n}_{2}<\mathrm{n}_{c}\right)$.

Here $A$ need not be small because $q_{11} \equiv\left(L_{\mathrm{II}} / \hbar\right)^{2 / 3} \sin ^{2} \theta$ can be of order unity. Since absorption cannot be ignored while calculating $B_{c} / B_{\infty}$ there is now no advantage in using equation (3). Hence we instead determine $R$ in (7) and then $A=1-|R|^{2}$.

Since the critical density lies above the step, (5) remains valid inside, the solution being again $B_{x} \propto A \mathrm{i}^{\prime}(-\xi)+h B \mathrm{i}^{\prime}(-\xi)$. Also, equation (8) is still valid for point 1. Above and near point 2 we may use a WKB approximation if $n_{c}$ is not close to $n_{2}$; hence

$$
B_{x} \propto \varepsilon^{1 / 4}\left[\exp \left(\mathrm{i} \int_{z_{2}}^{z} \varepsilon^{1 / 2} \mathrm{~d} z / \hbar\right)+r \exp \left(-\mathrm{i} \int_{z_{2}}^{z} \varepsilon^{1 / 2} \mathrm{~d} z / \hbar\right)\right] .
$$

Continuity of $\mathrm{d} \ln B_{x} / \mathrm{d} z$ at point 2 yields

$$
h=\left(\alpha_{2}^{\dagger}-r \alpha_{2}\right) /\left(-\beta_{2}^{\dagger}+r \beta_{2}\right)
$$

Use of (8) and (15) finally gives

$$
A=\frac{\left(1-|\rho|^{2}\right)\left(1-|r|^{2}\right)}{1+|\rho|^{2}-2|\rho r| \cos (\varphi+\psi)},
$$

where 


$$
|r| \mathrm{e}^{\mathrm{i} \varphi} \equiv r, \quad|\rho| \mathrm{e}^{\mathrm{i} \psi} \equiv \frac{\alpha_{1} \beta_{2}-\beta_{1} \alpha_{2}}{\alpha_{1} \beta_{2}{ }^{+}-\beta_{1} \alpha_{2}{ }^{\dagger}} .
$$

Here $|r|^{2}$ is the reflectivity of the critical region, clearly determined in Ginzburg's problem: $A_{G}\left(q_{\mathrm{II}}\right)=1-|r|^{2}$ (a simple, accurate approximation to $A_{G}$ is given in Appendix I). $|\rho|^{2}$ is the reflectivity of the step; for instance, for a density jump $\left(L_{\mathrm{l}} / \hat{\bar{t}} \rightarrow 0\right)$ we have

$$
|\rho|=\frac{\varepsilon_{1}^{1 / 2}-\varepsilon_{2}^{1 / 2}}{\varepsilon_{1}^{1 / 2}+\varepsilon_{2}^{1 / 2}},
$$

which is the usual Fresnel formula for normal incidence $(\theta$ having been neglected in the step). Note that if $L_{1} / \hbar \rightarrow \infty$, then we have $\xi_{1}, \xi_{2} \rightarrow \infty$ and thus $\alpha_{1} \beta_{2}-$ $\beta_{1} \alpha_{2} \rightarrow 0$, yielding $A=A_{G}\left(q_{\mathrm{II}}\right)$. Finally, the phase difference $\varphi$ between the WKB waves at $z_{2}^{+}$is given by

$$
\varphi \simeq 2 \int_{z_{2}}^{z_{i}} \varepsilon^{1 / 2} \mathrm{~d} z / \lambda+\left(\text { phase difference at } z_{c}^{-}\right) .
$$

The second term on the right goes from $-\pi / 2$ for $q_{\mathrm{II}} \ll 1$ to $\pi / 2$ for $q_{\mathrm{II}} \gg 1$ (GINZBURG, 1970), while the first one is roughly $4 \varepsilon_{2}^{3 / 2} L_{\mathrm{II}} / 3 \hat{\pi}$, a large quantity. The absorption $A$ in (16) is then a function of $\xi_{1}, \xi_{2}, q_{\mathrm{II}}$, and $L_{\mathrm{l}} / L_{\mathrm{IJ}}$. We may write these parameters in the form

$$
\begin{aligned}
& \xi_{1,2}=a_{\mathrm{I}}\left(n_{c}-n_{1,2}\right), \quad q_{\mathrm{I}, \mathrm{II}}=a_{\mathrm{I}, \mathrm{II}} n_{c} \sin ^{2} \theta \\
& L_{\mathrm{I}} / L_{\mathrm{II}}=\left(a_{\mathrm{I}} / a_{\mathrm{II}}\right)^{3 / 2}, \quad a \equiv \frac{\left(4 \pi \mathrm{e}^{2} / m_{e} c^{2}\right)^{1 / 3}}{(\mathrm{~d} n / \mathrm{d} z)^{2 / 3}} .
\end{aligned}
$$

If the phases are such that $\cos (\varphi+\psi)=1$, equation (16) gives

$$
A=1-\left(\frac{|\rho|-|r|}{1-|\rho r|}\right)^{2} .
$$

Thus, there will be total absorption if, in addition, $|\rho|=|r|$. Note that these are the usual conditions to get zero reflection of a wave propagating through two partly reflecting layers (the fact that the second one is here absorbing instead of transmitting makes no difference). Conditions $|\rho|=|r|$ and $\varphi+\psi=\operatorname{arc} \cos 1$ determine values of $n_{c} / n_{2}$ and $\theta$ in terms of $n_{1} / n_{2}, a_{1} n_{2}$, and $a_{\mathrm{I}} / a_{\mathrm{II}}$.

Note finally that absorption as found throughout this section is not really dependent on the profile being straight in region II: it just requires that $L_{\mathrm{II}} \gg \hat{\hbar}$, so as to make valid the WKB approximation there.

\section{ABSORPTION AT THE TOP CORNER}

The analysis of Section 2 predicts zero absorption when the critical density lies at the top of the step $\left(\varepsilon_{2}=0\right)$. For $\xi_{2} \rightarrow 0^{-}$in equation (13) we have $h \rightarrow 3^{-1 / 2}$ leading to $A=0$ in (10). For $\xi_{2} \rightarrow 0^{+}$in equation (17), $\alpha_{2}$ and $\beta_{2}$ become real so that $|\rho| \mathrm{e}^{\mathrm{i} \psi} \rightarrow 1$, leading to $A=0$ in (16). 
This result is not a trivial one. For a gentle, curved profile $n(z)$ the absorption, as function of incident wavelength, would be $A(\lambda) \simeq A_{G}(q)$ with $q \equiv(L / \hbar)^{2 / 3} \sin ^{2} \theta$ and $L(\hat{\pi}) \equiv n /(\mathrm{d} n / \mathrm{d} z)$ evaluated at the point where $n(z)=n_{c}(\hat{\pi})$. For $\mathrm{d} n / \mathrm{d} z$ positive and decreasing with increasing $n, q$ varies monotonically with $\lambda$ so that $A$ ( $\lambda$ could not present a minimum. The profile I-II of Section 2 is not gentle however, so that strong reflections could be expected to affect the absorption.

Actually equations (13) and (14) are invalid for $\varepsilon_{2}$ small, because, as already noticed, the WKB approximation of region II fails. To analyse the small $\varepsilon_{2}$ case in detail we define, for that region, $\eta \equiv-\varepsilon\left(L_{\mathrm{II}} / \hat{\pi}\right)^{2 / 3}$ and rewrite (2) in the form

$$
\frac{\mathrm{d}^{2}}{\mathrm{~d} \eta^{2}} \ln \left(\frac{B_{x}}{\eta^{1 / 2}}\right)+\left[\frac{\mathrm{d}}{\mathrm{d} \eta} \ln \left(\frac{B_{x}}{\eta^{1 / 2}}\right)\right]^{2}=\eta+q_{\mathrm{II}}+\frac{3}{4 \eta^{2}} .
$$

Also, to simplify the discussion, we here take $q_{\mathrm{Il}}$ to be large.

Consider first absorption in region I; then $\eta>\eta_{2}>0$ throughout II. Since equations (10) and (11) remain valid we just need to find $D$ in (12). For $\eta>\eta_{2} \gg$ $q_{\mathrm{ll}}^{-1 / 2}$, equation (20) becomes

$$
\left[\frac{\mathrm{d}}{\mathrm{d} \eta} \ln \left(\frac{B_{x}}{\eta^{1 / 2}}\right)\right]^{2} \simeq \eta+{ }^{\mathrm{q}}
$$

and thus, within the same approximation,

$$
\frac{\mathrm{d}}{\mathrm{d} \eta} \ln B_{x} \simeq-\left(\eta+q_{\mathrm{II}}\right)^{1 / 2}
$$

this is still a WKB solution, which is valid down to $\eta$ not too small because of the large value of $q_{11}$. For $\eta_{2} \ll q_{11}$ (a range that overlaps the previous one) the solution to equation (20) is still (22), if $\eta \gg q_{11}^{-1 / 2}$; if, on the other hand, $\eta_{2}<\eta \ll q_{\mathrm{Il}}$, then equation $(20)$ becomes

$$
\frac{\mathrm{d}^{2}}{\mathrm{~d} \eta^{2}} \ln \left(\frac{B_{x}}{\eta^{1 / 2}}\right)+\left[\frac{\mathrm{d}}{\mathrm{d} \eta} \ln \left(\frac{B_{x}}{\eta^{1 / 2}}\right)\right]^{2} \simeq q_{\mathrm{ll}}+\frac{3}{4 \eta^{2}} .
$$

The solution to this equation matching smoothly $(22)$ is

$$
\frac{\mathrm{d}}{\mathrm{d} \eta} \ln B_{x}=-q_{\mathrm{II}}^{1 / 2} \frac{K_{0}\left(q_{\mathrm{II}}^{1 / 2} \eta\right)}{K_{1}\left(q_{\mathrm{II}}^{1 / 2} \eta\right)},
$$

$K_{0}$ and $K_{1}$ being usual Bessel functions.

From equations (22) and (24) one gets

$$
D=-\sin \theta\left(1+\eta_{2} / q_{\mathrm{II}}\right)^{1 / 2}=-\left(\sin ^{2} \theta+\left|\varepsilon_{2}\right|\right)^{1 / 2}
$$

and

$$
D=-\sin \theta \times K_{0}\left(q_{11}^{1 / 2} \eta_{2}\right) / K_{1}\left(q_{11}^{1 / 2} \eta_{2}\right)
$$


respectively. For $\left|\varepsilon_{2}\right| \gg \sin ^{2} \theta$, equation (25) recovers (13). For $\lambda / L_{\mathrm{II}} \times$ $\sin \theta \ll\left|\varepsilon_{2}\right| \ll \sin ^{2} \theta$, one gets $D=-\sin \theta$ from either (25) or (26). Finally for $\left|\varepsilon_{2}\right|$ much less than $t / L_{\mathrm{II}} \sin \theta$, all the way down to zero, equation (26) yields

$$
D=-\left|\varepsilon_{2}\right| \frac{L_{11}}{\hbar} \sin ^{2} \theta \times \ln \left(\frac{2 \mathrm{e}^{-\gamma} \lambda / L_{11}}{\left|\varepsilon_{2}\right| \sin \theta}\right)
$$

where $\gamma=0.577 \ldots$ is Euler's constant. Using this value of $D$ in (11) yields $A \sim\left(\ln \left|\varepsilon_{2}\right|\right)^{-2}$ as $\varepsilon_{2} \rightarrow 0^{-}$.

Consider, next, absorption in region II, omitting details for brevity. We still have equations (8) and (15) so that $A=1-|R|^{2}$ again leads to (16). Now, however, $r$ is not a given function [equation (15) is here a definition of $r$ ] and is to be found from continuity of $\operatorname{d} \ln B_{x} / \mathrm{d} z$ at point 2 . When $\varepsilon_{2}$ is small enough equation (26) is still valid, but now $\eta_{2}$ is negative so that both $K_{0}$ and $K_{1}$ are complex. One finally finds that as $\varepsilon_{2} \rightarrow 0^{+}$both $\rho$ and $r$ approach unity and equation (16) yields $A \sim\left(\ln \varepsilon_{2}\right)^{-2}$.

The above shows that indeed $A$ vanishes at $n_{c}=n_{2}$. The vanishing is tied to the fact that $B_{c} \rightarrow 0$ as $n_{c} \rightarrow n_{2}$, a fact due to the slope discontinuity at point 2 (zero radius of curvature $\sigma$ there). If $\sigma$ is small but finite, $A\left(n_{c}\right)$ exhibits a minimum near $n_{c} / n_{2}=1, A_{\min } \sim(\ln \sigma)^{-2}$, which only vanishes in the limit $\sigma \rightarrow 0$ (App. II). Again, if $\sigma=0$ but a finite, though small, collision frequency $v$ is retained, $A$ presents a minimum near $n_{c} / n_{2}=1$.

To study this finite- $v$ case we obtain $B_{c} / B_{\infty}$, as previously, equating (6) and (7) at point 1 , leading to

$$
\frac{B_{c}}{B_{\infty}}=\frac{\varepsilon_{1}^{1 / 4}(1+R)^{\prime}}{A \mathrm{i}_{1}^{\prime}+h B \mathrm{i}_{1}^{\prime \prime}}\left(A \mathrm{i}_{0}^{\prime}+h B \mathrm{i}_{0}^{\prime}\right) \propto\left(1-3^{1 / 2} h\right)
$$

Next $h$ is found from (11) using equation (27), where here we let $n_{c} / n_{2} \rightarrow 1$ so that, from (1), $\left|\varepsilon_{2}\right|=v / \omega$. Then

$$
1-3^{1 / 2} h=\frac{2 A \mathrm{i}_{0}}{A \mathrm{i}_{0}^{\prime}} \frac{\left(L_{\mathrm{I}} / L_{\mathrm{II}}\right)^{1 / 3}}{q_{\mathrm{II}}}\left[\ln \left(\frac{\mathrm{e}^{\gamma /} v / \omega}{2 \hat{\mathrm{r} /} / L_{\mathrm{II}}} \sin \theta\right)\right]^{-1} .
$$

Hence, $B_{c} / B_{x} \sim(\ln v / \omega)^{-1}$ and $A \sim(\ln v / \omega)^{-2}$.

To conclude, we note, first, that retaining the small term $q_{1} B_{x}$ in equation (5) for region I does not modify the above results; in fact, if $v \rightarrow 0$ and $\sigma \rightarrow 0, A$ vanishes at $n_{c}=n_{2}$ for $q_{1}$ arbitrary (a case analysed in work to be published). Secondly, $A$ vanishes at point 1 too [as suggested by equation (10) for $\xi_{1} \rightarrow 0$ ]. However, a detailed analysis of absorption for $n_{\mathrm{c}} \rightarrow n_{1}^{-}$is not simple; furthermore, it is also useless for the application of interest considered in next section (see below).

\section{A PROFILE STEEPENED BY RADIATION PRESSURE}

We shall now apply the results of Section 2 to a particular density profile: the corona of expanding, stratified plasma that a solid target ejects when irradiated with a pulse of laser light; at high enough intensity the pressure of the radiation steepens 


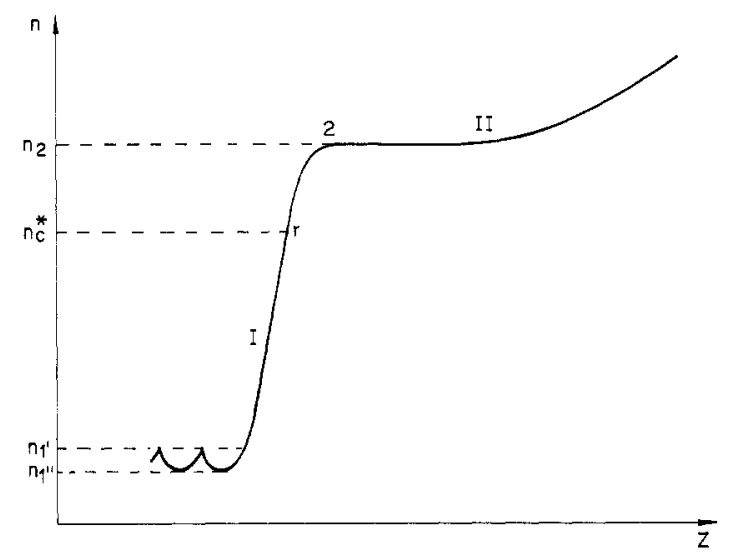

FIG. 2,- Plasma step produced by a high-intensity light of frequency $c / \hat{\kappa}^{*}$, normally incident on a solid target to the right; the steepening is due to its radiation pressure. Light is reflected at point $r$ in region I. The rippling below is due to the radiation pressure of the standing wave; the average density here decreases slowly to the left. Region II consists of a plateau and a gentle isothermal wave. $P$-polarized light of frequency $c / t i$ is shined on the profile at small angle $\theta$.

the plasma around the reflection point $r$ (Fig. 2) and produces a profile that is similar to the one discussed in Section 2; the rippling at the bottom of the step will be dealt with by using the mean $\left(n_{1^{\prime}}+n_{1^{\prime}}\right) / 2$ wherever a value of $n_{1}$ is necessary in the formulae of that section. The structure of the profile is well known for either normal incidence or s-polarization (Lee et al., 1977; Sanmartin and MONTAÑES, 1980a). Details convenient for our purposes are given in Appendix III for the case of normal incidence which, for simplicity, will be the one discussed here (we briefly comment on $s$-polarization at the end).

Neither case presents resonant absorption. Further, inverse bremsstrahlung is entirely negligible for such a thin region as that shown in Fig. 2. Hence, the electromagnetic wave will be a standing one there. Call $E_{\max }$ the last maximum of the electric field, before becoming evanescent, and $n_{c}^{*}$ and $c / t^{*}$ the critical density and the frequency of the light. We then have, first, $n_{1} / n_{c}^{*}=F_{1}\left(E_{\max }^{2} / n_{c}^{*} T_{e}\right)$ and $n_{2} / n_{c}^{*}=$ $F_{2}\left(E_{\max }^{2} / n_{c}^{*} T_{e}\right)$ for the densities at the ends of the step; and secondly, $\mathrm{d} n /\left.\mathrm{d} z\right|_{r}=$ $\left(n_{c}^{*} / \hat{\pi}^{*}\right) F_{3}\left(E_{\max }^{2} / n_{c}^{*} T_{e}\right)$ for the slope at point $r$, which we use to approximate the slope throughout the step. $T_{e}$ is the electron temperature and $F_{1}, F_{2}$ and $F_{3}$ are functions given in Appendix III. Finally, above the step, and for $T_{e}$ roughly constant in time, the profile is that of a gentle isothermal wave (following a plateau next to point 2), for which $n \propto \exp \left(z / c_{s} t\right)$; thus $\mathrm{d} n / \mathrm{d} z=n / c_{s} t$ in region II. Here $t$ is the time from the beginning of the pulse, $c_{s}$ is $\left(Z_{i} T_{e} / m_{i}\right)^{1 / 2}$, and $m_{i} / Z_{i}$ is the ion mass per unit charge.

Let now a pulse of $p$-polarized light be shined on the plasma as in Section 2 (this pulse will be assumed short compared with the main one, so that $t$ above is well defined). Using $n_{c} \hat{t}^{2}=n_{c}^{*} t^{* 2}$ and the previous results for $n_{1}$ and $n_{2}$, we obtain

$$
\begin{aligned}
& 1-\varepsilon_{1}=n_{1} / n_{c}=\left(\hat{\lambda} / \hat{\lambda}^{*}\right)^{2} F_{1}\left(E_{\max }^{2} / n_{c}^{*} T_{e}\right), \\
& 1-\varepsilon_{2}=n_{2} / n_{c}=\left(\hat{\lambda} / \hat{\hbar}^{*}\right)^{2} F_{2}\left(E_{\max }^{2} / n_{c}^{*} T_{e}\right) .
\end{aligned}
$$




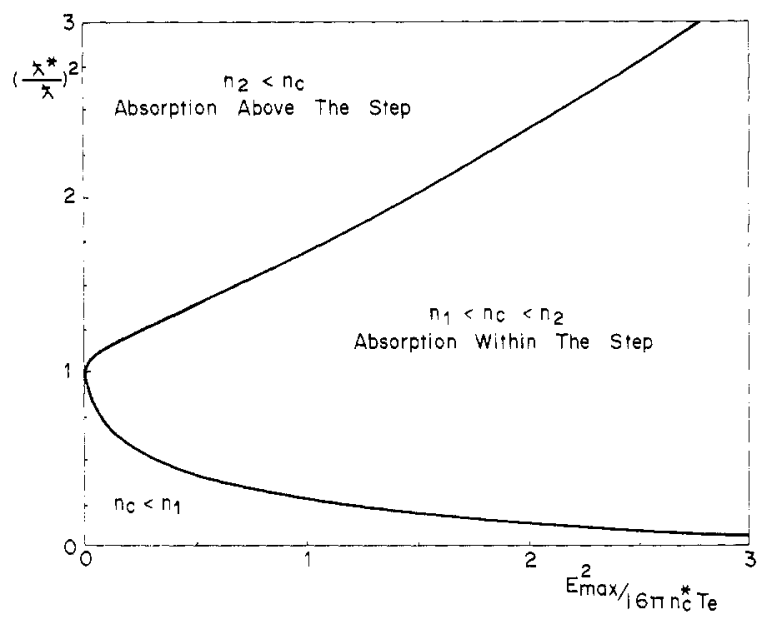

Fig. 3.--Parameter domains for absorption to occur in regions I or II of Fig. 2; the ordinate is the critical density ratio $\left(n_{c} / n_{c}^{*}\right)$ for probing and main light pulses, and the abscissa is a radiation to thermal pressure ratio ( $E_{\max }$ is the last maximum of the standing electric field). The lower and upper curves represent the functions $F_{1}$ and $F_{2}$ of equations (28) and (29).

These equations lead to Fig. 3, which shows the domains of the $\left(\hat{t}^{*} / \hat{t}\right)^{2}, E_{\mathrm{max}}^{2} / h_{c}^{*} T_{e}$ plane where conditions $n_{c}<n_{1}, n_{1}<n_{c}<n_{2}$, and $n_{2}<n_{c}$, apply; note that the lower and upper curves represent the functions $F_{1}$ and $F_{2}$ respectively. We also get

$$
L_{1} / \hat{\hbar}=\left(\hat{\lambda}^{*} / \hat{\lambda}\right)^{3} / F_{3}\left(E_{\max }^{2} / n_{c}^{*} T_{e}\right)
$$

Figure 4 shows $\left(L_{1} / \hat{x}\right)\left(\hat{\pi} / \hat{t}^{*}\right)^{3}$. Finally equation (10), with $h\left(\xi_{2}\right)$ given by (13) and

$$
\begin{gathered}
A_{\mathrm{G}} \simeq 2.64 q_{\mathrm{v}}, \quad q_{1} \simeq\left(\hat{\boldsymbol{t}}^{*} / \hat{t}\right)^{2} F_{3}^{-2 / 3} \sin ^{2} \theta \\
\xi_{1,2}=\left[\left(\hat{t}^{*} / \hat{x}\right)^{2}-F_{1,2}\right] F_{3}^{-2 / 3},
\end{gathered}
$$

yields the absorption in the range $n_{1}<n_{c}<n_{2} ; \theta$ being small, we have

$$
A=\theta^{2} \times \text { (function of } \hat{t}^{*} / \hat{t} \text { and } E_{\max }^{2} / n_{c}^{*} T_{e} \text { ). }
$$

Equation (16), giving the absorption in region II, simplifies considerably here. From (19) we obtain

$$
\mathrm{d} \varphi / \mathrm{d} t \propto c_{\mathrm{s}} / \bar{\pi}
$$

the proportionality constant being a function of $\hat{\pi}^{*} / \hat{\tau}$ and $E_{\max }^{2} / h_{c}^{*} T_{e}$, of order unity in general. Thus $\varphi$ changes rapidly with time; for instance, a change $\Delta \varphi=\pi$ requires typically a fraction of picosecond. Thus absorption measurements will usually average out the $\varphi$-dependence in (16). We then have

$$
A \simeq \bar{A} \equiv \int_{0}^{\pi} \frac{\mathrm{d} \varphi}{\pi} A(\varphi)=\frac{A_{G}\left(q_{\mathrm{II}}\right)}{1+\left(\rho^{2}-1\right)^{-1} A_{G}\left(q_{\mathrm{HI}}\right)},
$$




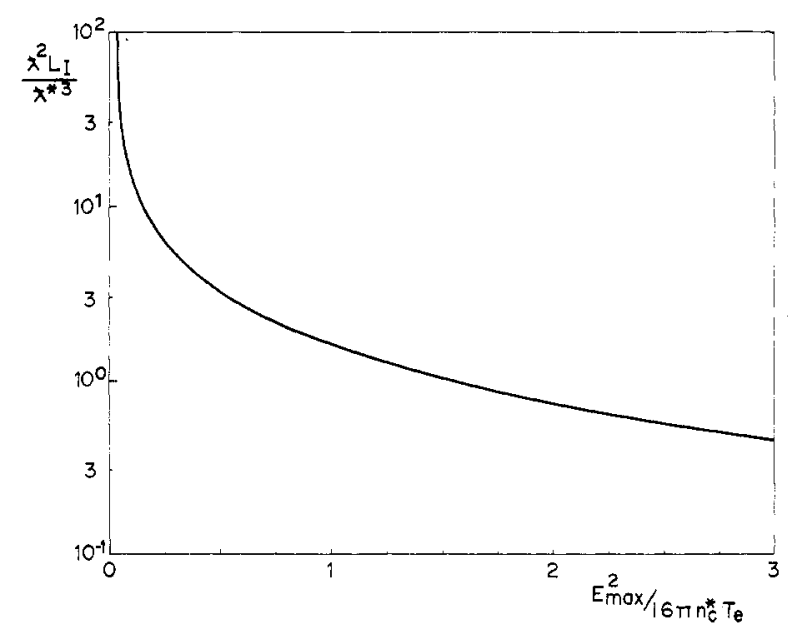

FIG. 4.-Normalized value of $L_{1} \equiv n_{\mathrm{c}} /(\mathrm{d} n / \mathrm{d} z)$ (at $n=n_{c}^{*}$ ) versus radiation to thermal pressure ratio.

where $\rho\left(\xi_{1}, \xi_{2}\right)$ is to be obtained from equation (17). Here a value for $L_{\text {II }}$ is required in $q_{\mathrm{II}} \equiv\left(L_{\mathrm{II}} / \hbar\right)^{2 / 3} \sin ^{2} \theta$ : since the slope to be used for absorption in region II is that at $n=n_{c}$, we get $\mathrm{d} n / \mathrm{d} z=n_{c} / c_{s} t$ or $L_{\mathrm{IJ}}=c_{s} t$. Thus, finally, for $n_{2}<n_{c}$,

$$
A=\text { function of } \dot{\pi}^{*} / \hat{\pi}, \quad E_{\max }^{2} / n_{c}^{*} T_{e}, \text { and }\left(c_{s} t / \tilde{\pi}^{*}\right)^{2 / 3} \theta^{2} .
$$

Equations (31) and (33) are easily extended to the case of a main pulse incident at angle $\theta_{s}$, and $s$-polarized; it just suffices to write $\lambda^{*} / \cos \theta_{s}$ for $\lambda^{*}$ in the equations. We also note that the rippling below point $1^{\prime}$ makes quite difficult the analysis of absorption at the bottom of the step, as suggested at the end of Section 3; the rippling does not impede, however, to relate $E_{\max }$ to the main pulse intensity, $I_{0}$, in the vacuum (Appendix III).

Figure 5 shows the absorption, given by equations (31) and (33), as function of $\hat{\pi}^{*} / \hat{z}$ for a few values of $E_{\text {max }}^{2} / n_{c}^{*} T_{e}$, a few values of $\left(c_{s} t / \hat{t}^{*}\right)^{2 / 3} \theta^{2}$ (range $n_{c}>n_{2}$ ) and $\theta=8^{\circ}$ (range $n_{1}<n_{c}<n_{2}$; here $A$ scales like $\theta^{2}$ ). Since the corner at the top of the step is rounded, equations (31) and (33) are not valid near $n_{c}=n_{2}$ (Section 3 and Appendix II); the minimum of $A$ is not zero and is here left undetermined, as indicated in the figure. The failure of the results for $n_{c} \leqslant n_{1}$ is indicated in the figure too.

\section{DISCUSSION}

We have studied how resonant absorption of $p$-polarized light, shined at a small angle $\theta$ on a collisionless step-profile plasma, depends on the critical density $n_{c}(t)$ for the light. We found that above the step the absorption is total if $n_{c}$ and $\theta$ take particular values corresponding to equal reflectivities of both step and region around critical, and to some appropriate phase condition.

We also found that as $n_{c}$ crosses the top of the step $A$ goes through a minimum. For the case of Fig. 1, which has a slope discontinuity (that is, zero radius of curvature $\sigma$ ) at point 2, that minimum vanishes. With growing $\sigma$ the minimum fills up, 


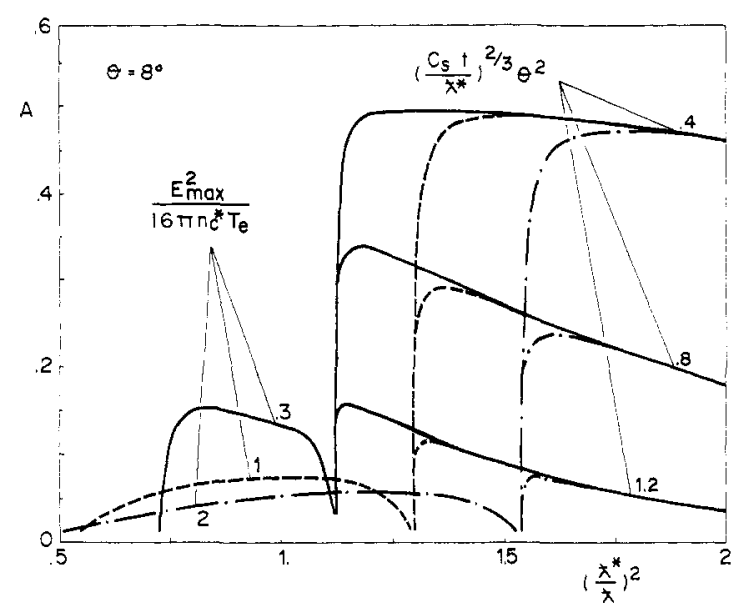

FiG. 5.-Absorption A of $p$-polarized light in the profile of Fig. 2, taken from equations (31) and (33). The minima of $A$ occur at $n_{c} \simeq n_{1}$ and $n_{c} \simeq n_{2}$, and their values are left undetermined. Between minima [equation (31)] the curves drawn correspond to $\theta=8^{\circ}$, A scaling like $\theta^{2}$.

disappearing at about $10 \%$ of $\left(n_{2}-n_{1}\right) /(\mathrm{d} n / \mathrm{d} z)$, although a precise condition depends on local details of the profile. If the slope is discontinuous but collisions, though unfrequent, are considered, that minimum is again non-zero.

For the case of Fig. 1 the reflectivity $|\rho|^{2}$ of the step itself goes to unity as $n_{c} \rightarrow n_{2}$, suggesting an evanescent field behind. Indeed in the limit of a density discontinuity the reflectivity is given by Fresnel formula for normal incidence, equation (18) $(\theta$ was neglected in the analysis of the step), which for $\varepsilon_{2}=1-n_{2} / n_{c} \rightarrow 0$ gives $|\rho|=$ 1 ; as well known the field behind a dielectric discontinuity is not zero even with total reflection.

Since resonant absorption always occurs in a region of evanescent field, the fact that $|\rho| \rightarrow 1$ as $n_{2} \rightarrow n_{c}$ need not imply null absorption. Further, if the small value of $\theta$ is retained in the analysis of the fields within the step, it is found that there is absorption for $n_{c}=n_{2} / \cos ^{2} \theta$ (a value then leading to $|\rho|=1$ ) and there is not for $n_{c}=n_{2}$. The fact is that resonant absorption is proportional to the square of the magnetic field at the critical surface, and this field, we find, cannot but vanish when that surface lies at a sharp turn of the density slope.

We applied our results to the step profile produced by irradiating a solid target with a high-intensity laser pulse that steepens the plasma by radiation pressure; this main pulse, of frequency $c / \hbar^{*}$, was taken to be normally incident, though results are easily extended to a $s$-polarized pulse. The absorption $A$ of probing $p$-polarized light, shined on the plasma, is given in Fig. 5, and depends on $E_{\max }^{2} / n_{c}^{*} T_{e}, \vec{t}^{*} / \vec{\pi}$, and $\theta$ (within the step) or $\left(c_{s} t / t^{*}\right)^{2 / 3} \theta^{2}$ (above the step). $E_{\max }$ is the last maximum of the electric field just before becoming evanescent, $c_{s}$ is the ion-acoustic speed, $T_{e}$ the electron temperature, and $t$ the time from the beginning of the main pulse. The change of $A$, and its minimum, around $n_{c}=n_{2}$, is dependent on the ratio $E_{\text {max }}^{2} / n_{\mathrm{c}}^{*} T_{e}$, and could be used to determine $T_{e}$. 


\section{REFERENCES}

Aliev Y. M., Gradov O. M., Kyrie A. Y., Cadez V. M. and Vukovic S. (1977) Phls. Rel. A $15,2120$. ANiosiewicz H. A. (1964) Handbook of Mathematical Functions (edited by M. ABRAMowitz and I. A. STEGUN) p. 435. NBS Applied Maths Series 55, Washington.

Forslund D. W., Kindel J. M., Lee K., Lindmax E. I. and Morse R. L. (1975) Phys. Ret. A 11, 679.

Ginzburg V. L. (1970) The Propagation of Electromagnetic Waves in Plasmas. Section 20. Pergamon. Oxford.

Kindel J. M., Lee K. and Lindman E. L. (1975) Phy's. Ret. Lett. 34, 134.

Kull H. J. (1983) Phy:sics Fluids 26, 1881.

LANDAU L. D. and Lifshitz E. M. (1960) Electrodynamics of Continuous Media, Sections 61 and 68 , Pergamon, Oxford.

Lee K., Forsllnd D. W., Kindel J. M. and Lindman E. L. (1977) Physics Fluids 20, 51.

Nicolas J. A. and Sanmartix J. R. (1985) Plasma Physics 27, 279.

Sa.vmartin J. R. and Montañes J. L. (1980a) Phy:sics Fluids 23, 2413.

Sanmartin J. R. and Montañes J. L. (1980b) J. Plasma Phys. 23, 349

\section{A P PENDIX I}

A very simple and accurate analytical expression for $A_{\mathrm{G}}(q)$ for use in equations (16) and (33), is

$$
A_{G}(q) \simeq \frac{2 a q}{2+a q} \exp \left(-\frac{4}{3} q^{3 / 2}\right), \quad a \equiv 4 \pi^{2}\left|A \mathrm{i}_{0}^{\prime}\right|^{2} \simeq 2.64 .
$$

It behaves correctly at small $q\left(A_{G} \simeq a q\right)$ and large $q\left[A_{G} \simeq 2 \exp \left(-4 q^{3 / 2} / 3\right)\right]$, and has a single maximum of 0.499 at $q \simeq 0.460$, values indistinguishable from corresponding results obtained with a numerical solution of the wave equation (ForSLUND et al., 1975). Figure 6 shows the general agreement with such a solution.

\section{A PPENDIX II}

We here assume that the head of the step of Fig. 1 is rounded. As in Section 3 we take for simplicity $q_{\mathrm{II}}$ large; we also take $L_{\mathrm{I}} / \hbar$ large (though retaining $q_{1}$ small). Within and in the corner itself (Fig. 7 ) $A$ is given by the expression (10) times the factor $L_{c} / L_{1}$, that accounts for the turn of the slope in the corner; in going around it, $L_{c}$, which is $\mathrm{d} z /\left.\mathrm{d} \ln \right|_{n=n_{c}}$, grows from $L_{\mathrm{I}}$ to $L_{\mathrm{II}}$.

Since $\xi_{1} \gg 1$ (except for $n_{c}$ near $n_{1}$ ) we have

$$
\frac{A}{A_{G}\left(q_{\mathrm{I}}\right)}=\frac{\left(1-3^{1 / 2} h\right)^{2}}{1+h^{2}} \frac{L_{\mathrm{c}}}{L_{1}}
$$

For $n_{c}$ not too close to $n_{2}, A / A_{G}\left(q_{1}\right)$ is unity; note that $h$ is then given by (11) and is negligible because $\left|\zeta_{2}\right|$ is large. Since $q_{\mathrm{I}}$ is nearly constant throughout the corner, to obtain the minimum absorption it suffices to study how, the right-hand side of (II.1) drops below unity inside the corner, when $\varepsilon_{2}\left(n_{c}\right) \equiv 1-n_{2} / n_{c}$ is varied $\left(\hat{t}, L_{1}, L_{\mathrm{ll}}\right.$, and $q_{\mathrm{l}}$, may be taken constant as $q_{\mathrm{l}}$ ).

We consider a profile locally given by

$$
\frac{n}{n_{2}}=1+\frac{L_{11}+L_{1} z}{L_{11} L_{1}} \frac{z}{2}-\left[\left(\frac{L_{11}-L_{1} z}{L_{11} L_{1}} \frac{z}{2}\right)^{2}+\tau^{2}\right]^{1 / 2} .
$$

The parameter $\tau$ is related to the minimum radius of curvature $\sigma$ in the plane $n / n_{2}, z / L_{1}(\tau \simeq \sigma / 5.3)$. As $\tau \rightarrow 0$ the profile of Fig. 1 is recovered; we shall assume that $\tau \ll\left(L_{\mathrm{I}} / L_{\mathrm{II}}\right)^{1 / 2}$ and $\tau \sin \theta \ll L_{\mathrm{I}} / L_{\mathrm{II}}$. We called $n_{2}$ the density where the two asymptotes $\left(n / n_{2}=1+z / L_{1}\right.$, or $\left.1+z / L_{\mathrm{II}}\right)$ meet, having set $z=0$ there.

From equation (II.2) one obtains

$$
\frac{L_{c}}{L_{\mathrm{l}}}=\frac{p+1}{p+L_{\mathrm{l}} / L_{\mathrm{Il}}}
$$

where

$$
p\left(n_{c}\right)=\frac{L_{\mathrm{I}}}{L_{11}}+\frac{\varepsilon_{2}^{2}}{2 \tau^{2}}-\frac{\varepsilon_{2}}{\tau}\left(\frac{L_{1}}{L_{\mathrm{II}}}+\frac{\varepsilon_{2}^{2}}{4 \tau^{2}}\right)^{1 / 2} .
$$

To next obtain $h\left(n_{c}\right)$ we integrate the exact equation for $B_{x}$ between $z_{a}$ and $z_{b}$, just outside the corner (Fig. 7), though satisfying $\left|z_{a}\right| \gg \tau L_{\mathrm{I}}, z_{b} \gg \tau\left(L_{\mathrm{l}} L_{\mathrm{II}}\right)^{1 / 2}$ : 


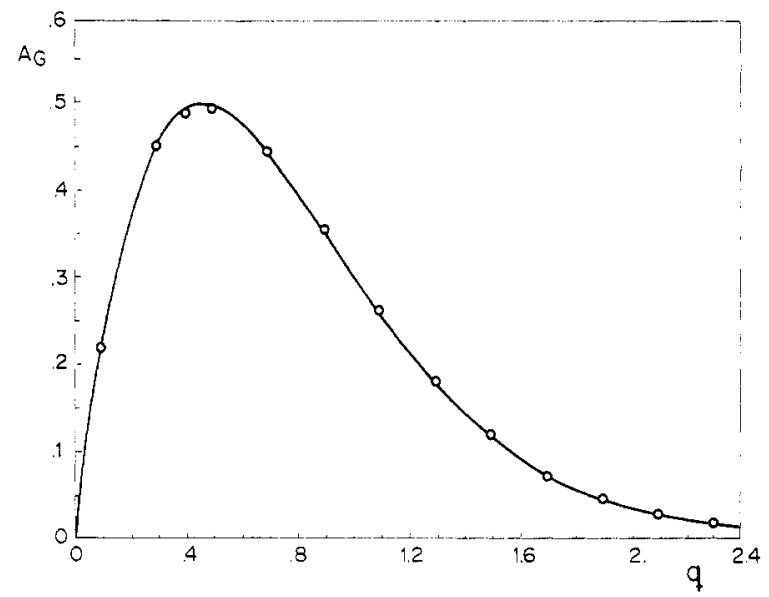

FIG. 6.--Ginzburg's absorption function $A_{G}(q)$ from the approximate formula in Appendix I (continuous line), and from numerical calculations (data points, ForsLund at al., 1975).

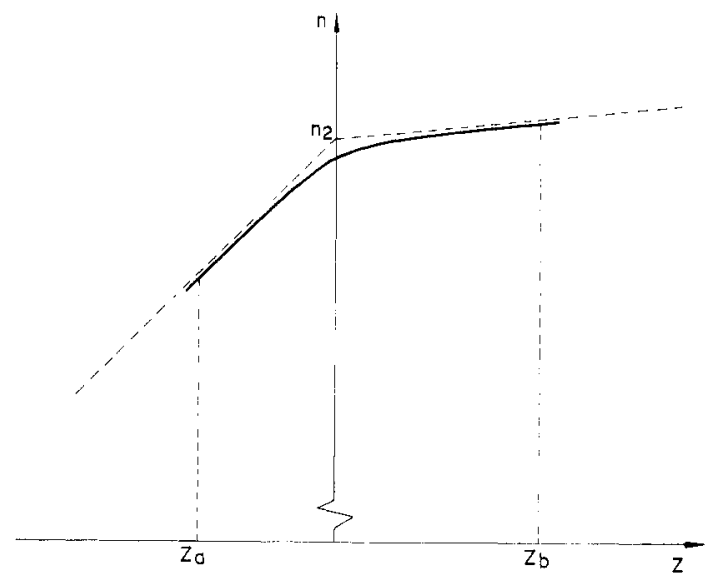

FIG. 7.-Head of the step in Fig. 1, for a small but finite radius of curvature. For $z<z_{a}$ and $z>z_{b}$ the profile is assumed straight and the field is given by equations (6) and (24) respectively.

$$
\left.\frac{\dot{\hbar} \mathrm{d} B_{x}}{\varepsilon}\right|_{a} ^{b}=\int_{a}^{b} B_{x} \frac{\mathrm{d} z}{\hat{\hbar}} \frac{\sin ^{2} \theta}{\varepsilon}-\int_{a}^{b} B_{x} \frac{\mathrm{d} z}{\dot{\gamma}} .
$$

We may set $B_{x} \simeq B_{c}$, neglect the last integral, and write the first one as $B_{i} I \sin ^{2} 0$. Using equations (6) and (24) for $z \leqslant z_{a}$ and $z \geqslant z_{b}$ respectively and a small argument expansion of all Bessel functions, equation (II.5) becomes

$$
\begin{aligned}
& -\sin ^{2} \theta \frac{L_{\mathrm{II}}}{i} \ln \left(\frac{L_{\mathrm{II}}}{\pi} \frac{\mathrm{e}_{r}^{\prime}}{2}\left|\varepsilon_{b}\right| \sin \theta\right)+\left(\frac{L_{1}}{\bar{t}}\right)^{1 / 3} \frac{A \mathrm{i}_{0}}{A \mathrm{i}_{0}^{\prime}} \frac{1+3^{1 / 2} h}{1-3^{1 / 2} h}=I \sin ^{2} \theta . \\
& I \simeq \frac{L_{11}}{i}\left(\frac{p-L_{\mathrm{l}} / L_{\mathrm{ll}}}{p+L_{\mathrm{l}} / L_{\mathrm{ll}}} \frac{\ln p}{2}+\frac{L_{\mathrm{l}} / L_{\mathrm{l}}}{p+L_{\mathrm{l}} / L_{\mathrm{ll}}} \ln \frac{L_{\mathrm{l}}}{L_{\mathrm{ll}}}+\ln \frac{\tau}{\left|\varepsilon_{b}\right|}-\mathrm{i} \pi \frac{L_{c}}{L_{\mathrm{ll}}}\right) .
\end{aligned}
$$


Using the above equations one obtains $A / A_{G}\left(q_{\mathrm{l}}\right)$ as a function of $\varepsilon_{2}\left(n_{c}\right), \tau, L_{\mathrm{l}} / L_{\mathrm{ll}}, L_{\mathrm{II}} / \hbar$ and $q_{\mathrm{II}}$. A minimum exists for $p \gg L_{\mathrm{I}} / L_{\mathrm{II}}$

$$
\frac{A_{\min }}{A_{G}\left(q_{1}\right)} \simeq \frac{5.6\left(L_{\mathrm{I}} / L_{\mathrm{II}}\right)^{2 / 3}}{q_{\mathrm{I}}^{2} p_{m}\left(1+p_{m}\right)}
$$

where

$$
2\left(1+p_{m}\right)+\ln p_{m} \simeq \ln \left[\frac{2}{\mathrm{e}^{\gamma}} \frac{\left(\hat{\kappa} / L_{\mathrm{II}}\right)^{4 / 3}}{q_{\mathrm{II}} \tau^{2}}\right]
$$

hence $A_{\min } \sim 1 / p_{m}^{2} \sim \mid \ln \sigma^{-2}$. For $\tau \simeq \sigma / 5.3=0.01, L_{\mathrm{I}} / L_{\mathrm{II}} \simeq 0.02, L_{\mathrm{l}} / \hbar=2$, and $q_{\mathrm{II}}=2$, we get $A_{\text {min }} \simeq 0.12 A_{G}\left(q_{1}\right)$.

\section{APPENDIX III}

Here we briefly recall some features of the structure in Fig. 2 for normal incidence (LEE et al., 1977; SANMARTIN and MONTAÑES, 1980a). The structure is determined by conservation laws for mass and momentum, an uniform temperature $T_{e}$, and the wave equation, and is characterized by a single parameter, $E_{\max }^{2} / n_{c}^{*} T_{e}$. This ratio measures the importance of radiation pressure against thermal pressure; $E_{\max }$ is the electric field at the last maximum (point $1^{\prime}$ ) of the standing wave (absorption being negligible in the thin region of interest).

Let $M$ be the plasma-flow Mach number at any point in the region, in a frame moving with the step. We have

$$
g\left(M_{2}\right)=E_{\max }^{2} / 8 \pi n_{c}^{*} T_{e}, \quad M_{2}<1
$$

where $n_{c}^{*}$ is the critical density for the incident light, and

$$
g(M)=M^{2}-\ln M^{2}-1
$$

then, defining $2 n_{1} \equiv n_{1}+n_{1^{\prime \prime}}$, we have

$$
\begin{aligned}
& n_{1} / n_{c}^{*}=\left(1+M_{1}^{\prime \prime}\right)\left(M_{2} g\right) / 4 M_{1}\left(1-M_{2}\right)^{2} \equiv F_{1}\left(E_{\max }^{2} / n_{c}^{*} T_{e}\right), \\
& n_{2} / n_{c}^{*}=g\left(M_{2}\right) / 2\left(1-M_{2}\right)^{2} \equiv F_{2}\left(E_{\max }^{2} / n_{c}^{*} T_{e}\right)
\end{aligned}
$$

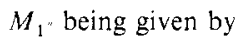

$$
g\left(M_{1}^{\prime \prime}\right)=g\left(M_{2}\right), \quad M_{1 "}>1
$$

At the reflection (critical) point $r$, where $n=n_{c}^{*}$, we also have

$$
\left.\frac{\lambda^{*}}{n_{c}^{*}} \frac{\mathrm{d} n}{\mathrm{~d} z}\right|_{r}=\left(\frac{g\left(M_{2}\right)-g\left(M_{r}\right)}{1-M_{r}^{2}}\right)^{1 / 2}\left(\frac{g\left(M_{r}\right)}{1-M_{r}^{2}}-2\right)^{1 / 2} \equiv F_{3}\left(E_{\text {mix }}^{2} / n_{c}^{*} T_{e}\right) ;
$$

here

$$
M_{r}=M_{2} g\left(M_{2}\right) / 2\left(1-M_{2}\right)^{2} .
$$

$E_{\text {max }}$ is related to the intensity of the light incident from the vacuum, $I_{0}$. For the values of interest $\left(I_{0}>10^{15} \mathrm{~W} \mathrm{~cm}^{-2}\right.$ for $)=1.06 \mu \mathrm{m}$, say) absorption, due to inverse bremsstrahlung, is small throughout (Nicolas and SANMARTin, 1985). In a rough approximation one may neglect it and use a WKB analysis that averages the underdense rippling, to obtain (LEE el al., 1977)

$$
\frac{l_{o}}{c n_{c}^{*} T_{c}}=\frac{E_{\max }^{2}}{32 \pi n_{c}^{*} T_{e}}\left(1-\frac{n_{1}}{n_{c}^{*}}\right)^{1 / 2} \text {. }
$$

A formulation that takes into account the rippling was given by SANMARTIN and MONTAÑES (1980b); at the lowest intensities to consider, differences are about $10 \%$. 